\title{
Many-body interactions among adsorbed atoms and molecules within carbon nanotubes and in free space
}

\author{
Milen K. Kostov*, Milton W. Cole, and John Courtenay Lewis \\ Department of Physics, \\ The Pennsylvania State University, University Park, PA 16802, USA \\ Phong Diep and J. Karl Johnson \\ Department of Chemical and Petroleum Engineering, \\ The University of Pittsburgh, Pittsburgh, PA 15261, USA
}

October 25, 2018

\begin{abstract}
Studies of three-dimensional and two-dimensional condensed phases have shown that manybody interactions contribute $\sim 10 \%$ to the equations of state of noble gases. This paper assesses the importance of three-body triple dipole interactions for quasi-one-dimensional phases of $\mathrm{He}, \mathrm{Ne}, \mathrm{H}_{2}$, Ar, Kr and Xe confined within interstitial channels or on the external surfaces of nanotube bundles. We find the substrate-mediated contribution to be substantial: for interstitial $\mathrm{H}_{2}$ the well depth of the effective pair potential is reduced to approximately one half of its value in free space.

We carry out $a b$ initio calculations on linear and equilateral triangular configurations of $\left(\mathrm{H}_{2}\right)_{3}$ and find that overlap interactions do not greatly change the DDD interaction in the linear configuration when the lattice spacing is greater than about $3 \AA$. However, the DDD interaction alone is clearly insufficient for the triangular configurations studied.
\end{abstract}

\section{Introduction}

Adsorbates within carbon nanotubes and bundles of nanotubes have been the focus of much recent attention (see for example refs. [1]-[8]). In particular, novel quasi-one dimensional adsorbate phases have been predicted. The closest to true one-dimensional behavior is likely to be realized by small atoms or molecules ( $\mathrm{He}, \mathrm{Ne}, \mathrm{H}_{2}$ ) in the "interstitial channel" (IC) phase, and the "groove" phase. For the IC phase, the molecules adsorb in channels bounded by three contiguous nanotubes within bundles. The groove phase comprises adsorbate in the grooves between adjacent tubes on the perimeter of a nanotube bundle. In both of these phases the molecules are confined transversely but can move more or less freely along the lengths of the nanotubes.

To date, in exploring adsorption on and in nanotubes, relatively little attention has been paid to the detailed nature of the interactions among adsorbate molecules. Indeed, most studies have assumed that the pairwise interaction does not differ significantly from its free-space form. In this paper we show that, for the IC and groove systems considered, the proximity of a polarizable medium, the nanotube walls, will profoundly alter the effective pair potential between two adsorbate molecules, to the extent of producing qualitative changes in expected behavior.

In two- and three-dimensional systems many-body effects have been extensively studied [9, 10, 11. Barker [10], in particular, showed that equilibrium properties of condensed rare gas systems could be obtained accurately using potentials in which the pair potentials were obtained from gas-phase data, and the higher-order contributions were purely of Axilrod-Teller-Muto or "triple-dipole" (DDD) type, even though this is exact only asymptotically for large molecular separations. Barker argued that extensive

\footnotetext{
*corresponding author, e-mail: mkk143@psu.edu, fax:814-865-3604
} 
cancellation must take place among other many-body interactions, at least one of which is known to be comparable in magnitude to the DDD interaction.

Analogously in two dimensions, adsorbed monolayer films manifest two types of many-body contributions that are relevant to quasi-one-dimensional nanotube phases. One is that involving three adatoms. The other is a modification of the adatom pair potential by the substrate acting as a third body. McLachlan derived an asymptotic expression, valid at large adatom separation, for this substrate mediation energy [12]. As in the three-dimensional case, experimental data at condensed phase densities in adsorbate films are consistent with the asymptotic expression [13, 14]. However, the experimental data for two-dimensional films are too limited to provide a thorough test of the adequacy of McLachlan's interaction. We note that a third three-body energy in films involves two substrate atoms and an adatom. This oft-neglected interaction can make a substantial correction to the gas-surface adsorption potential.

While extensive cancellation occurs as discussed above in three dimensions, among many-body interactions other than the DDD three-body interaction, this cannot be assumed a priori in one dimension because of the strong angular dependence typical of many-body interactions. We have therefore extended a recent accurate $a b$ initio calculation of the potential energy surface of $\mathrm{H}_{2}-\mathrm{H}_{2}$ [15 to linear equispaced $\left(\mathrm{H}_{2}\right)_{3}$ and to $\left(\mathrm{H}_{2}\right)_{3}$ arranged in equilateral triangles. While significant deviations from the DDD interaction are found for the equilateral triangular configurations, the deviations from DDD for linear $\left(\mathrm{H}_{2}\right)_{3}$ are important only for separations small compared to the equilibrium separation of $\mathrm{H}_{2}-\mathrm{H}_{2}$.

In this paper we consider a linear array of rare gas atoms or of $\mathrm{H}_{2}$, henceforth denoted $\mathrm{A}$, each separated from its nearest neighbor by a lattice constant $a$. In Section 2 we evaluate the net DDD contribution arising from interactions among all sets of triplets AAA. The resulting three-body energy per atom, which we call $E_{A A A}^{(3)}$, turns out to be negative and of order $1 \%$ of the total pair interaction energy. We next evaluate an energy per pair $V_{A A C}^{(3)}$, due to a summation of the DDD interactions between two A particles and all $\mathrm{C}$ atoms. This energy is found to be positive and of large magnitude, equal approximately to half of the interaction between the atoms in free space. When $V_{A A C}^{(3)}$ is added to the free space interaction, there results an effective pair potential which has a well depth significantly smaller than the free space well depth. This reduced attraction implies that collective phenomena such as condensation, ought to occur at much lower temperatures than would be predicted if only the free-space interactions were taken into account, and substrate mediation were ignored. Qualitatively similar behavior is derived as a consequense of the DDD interaction for $\mathrm{He}, \mathrm{Ne}, \mathrm{H}_{2}, \mathrm{Ar}, \mathrm{Kr}$ and $\mathrm{Xe}$ atoms/molecules adsorbed into the grooves of a nanotube bundle. In Section 3 we discuss the ab initio calculation carried out on $\left(\mathrm{H}_{2}\right)_{3}$ in linear and triangular arrays.

While this paper evaluates electronic substrate mediation of adsorbate interactions, a substrate phonon mediation mechanism also exists. Such phonon mediation effects on adatom interactions on planar surfaces are reviewed by Gottlieb and Bruch [14]. While no such evaluation has been performed for atoms or molecules in nanotubes, the analogous effect of nanotube phonons on the electron-electron interaction has recently been assessed by Woods and Mahan [16].

\section{Adsorbates in IC and Groove Phases}

We begin by considering particles A confined to the axis of an IC phase and separated by a lattice constant $a$. There are two relevant three-body energies which we consider. The first is the net DDD energy arising from interactions among all sets of triplets on the line. The second is the total DDD interaction involving an adatom pair and all possible $\mathrm{C}$ atoms. The potential energy $\Phi$ corresponding to a linear array of $N$ particles specified by their position vectors $\overrightarrow{r_{1}}, \ldots, \overrightarrow{N_{N}}$ is given by

$$
\Phi\left(\overrightarrow{r_{1}}, \ldots, \overrightarrow{r_{N}}\right)=\sum_{i<j}^{N} U_{A A}^{(2)}\left(\overrightarrow{r_{i}}, \overrightarrow{r_{j}}\right)+\sum_{i<j<k}^{N} U_{A A A}^{(3)}\left(\overrightarrow{r_{i}}, \overrightarrow{r_{j}}, \overrightarrow{r_{k}}\right)+\sum_{i<j<k}^{N} U_{A A C}^{(3)}\left(\overrightarrow{r_{i}}, \overrightarrow{r_{j}}, \overrightarrow{r_{k}}\right),
$$

where $U^{(2)}$ and $U^{(3)}$ are pair and DDD triplet potential functions and four-body (and higher order) interactions are ignored. For purposes of estimating the total pair energy we will temporarily assume

\footnotetext{
${ }^{1}$ In this paper we do not consider the implications of substrate-mediated adsorbate interactions for condensation involving interactions among different IC or groove phases in a nanotube bundle (see e.g. Ref.|8]).
} 
that the pair potential $U_{A A}^{(2)}\left(\overrightarrow{r_{i}}, \overrightarrow{r_{j}}\right)$ is isotropic and of Lennard-Jones 12-6 form with distance parameter $\sigma$ and energy parameter $\epsilon$. The total pair interaction energy per particle A can be written as

$$
E_{A A}^{(2)}=\frac{V_{A A}^{(2)}}{N}=4 \epsilon\left[\left(\frac{\sigma}{a}\right)^{12} \zeta(12)-\left(\frac{\sigma}{a}\right)^{6} \zeta(6)\right],
$$

in the limit $N \rightarrow \infty$, where $\zeta$ is the Riemann zeta function. The right-hand side of Eq. (2) has a minimum at $a / \sigma=1.122$. Evaluating Eq. (2) at that minimum we obtain:

$$
E_{A A}^{(2)}=\frac{V_{A A}^{(2)}}{N}=-1.035 \epsilon=-0.509 \frac{C_{6}}{a^{6}},
$$

where $C_{6}=4 \epsilon \sigma^{6}$. Next we assess the contribution of the DDD interactions $U_{A A A}^{(3)}$ to $V_{A A}^{(2)}$. A simple result for $U_{A A A}^{(3)}$ holds for isotropic oscillators, at large separations:

$$
U_{A A A}^{(3)}=\nu_{A A A} \frac{3 \cos \left(\theta_{i}\right) \cos \left(\theta_{j}\right) \cos \left(\theta_{k}\right)+1}{r_{i j}^{3} r_{i k}^{3} r_{j k}^{3}},
$$

where $\nu_{A A A}$ is the triple dipole dispersion energy coefficient, $r_{i j}, r_{i k}, r_{j k}$ are the interatomic distances in a given triplet, while $\theta_{i}, \theta_{j}$ and $\theta_{k}$ are the internal angles of the triangle formed by the atoms $i, j$ and $k$. The sign of the triple dipole energy depends on the geometry of the triangle formed by the three atoms. Indeed, the DDD term is positive for acute triangles, while for most obtuse triangles it is negative. In particular, $U_{A A A}^{(3)}$ is negative for the linear configuration of adsorbate particles, since $\cos \left(\theta_{i}\right) \cos \left(\theta_{j}\right) \cos \left(\theta_{k}\right) \simeq-1$ in that case. Then, the net DDD potential becomes:

$$
V_{A A A}^{(3)} \cong-\sum_{i<j<k}^{N} \frac{2 \nu_{A A A}}{\left(r_{i j} r_{i k} r_{j k}\right)^{3}} .
$$

Summing over all possible triplets we obtain the net DDD energy per atom:

$$
\begin{gathered}
E_{A A A}^{(3)}=-\frac{2 \nu_{A A A}}{a^{9}}\left[\frac{1}{2^{3}} \zeta(9)+2 \sum_{n=2}^{\infty} \sum_{m=1}^{\infty} \frac{1}{m^{3}(n+m-1)^{3}(n+2 m-1)^{3}}\right], \\
E_{A A A}^{(3)}=-\frac{0.27 \nu_{A A A}}{a^{9}} .
\end{gathered}
$$

The coefficient $\nu_{A A A}$ can be estimated from a Drude model of isotropic oscillators from the coefficient $C_{6}: \nu_{A A A}=\frac{3}{4} C_{6} \alpha_{A}$, where $\alpha_{A}$ is the static polarizability of the adatom. Then the ratio of the DDD energy to the pair interaction energy is:

$$
\frac{E_{A A A}^{(3)}}{E_{A A A}^{(2)}} \cong 0.4 \frac{\alpha}{a^{3}} .
$$

This ratio is very small $\left(0.8 \%, 0.3 \%\right.$ and $0.5 \%$ for $\mathrm{H}_{2}$, He and Ne, respectively, using values of $\alpha$ and $a$ taken from [1]).

We next evaluate and assess the net DDD interaction involving the adatom pair and all possible $\mathrm{C}$ atoms. The effective pair potential has the form:

$$
V_{\mathrm{eff}}^{(2)}\left(\overrightarrow{r_{i}}, \overrightarrow{r_{j}}\right)=U_{A A}^{(2)}\left(\overrightarrow{r_{i}}, \overrightarrow{r_{j}}\right)+V_{A A C}^{(3)}\left(\overrightarrow{r_{i}}, \overrightarrow{r_{j}}\right)
$$

For a linear configuration of adsorbate particles: $U_{A A}^{(2)}\left(\overrightarrow{r_{i}}, \overrightarrow{r_{j}}\right)=U_{A A}^{(2)}\left(\left|z_{i}-z_{j}\right|\right)$ and $V_{\text {eff }}^{(2)}\left(\overrightarrow{r_{i}}, \overrightarrow{r_{j}}\right)=$ $=V_{\text {eff }}^{(2)}\left(\left|z_{i}-z_{j}\right|\right)$, where $z$ axis is along the axis of the IC. The DDD energy $V_{A A C}^{(3)}$ is given by:

$$
V_{A A C}^{(3)}\left(\overrightarrow{r_{i}}, \overrightarrow{r_{j}}\right)=\sum_{k} U_{A A C}^{(3)}\left(\overrightarrow{r_{i}}, \overrightarrow{r_{j}}, \overrightarrow{r_{k}}\right)
$$


Here $\overrightarrow{r_{k}}$ is the position of the $k^{t h}$ carbon atom along the nanotube surface and $U_{A A C}^{(3)}$ is its DDD interaction with the adatoms at $\overrightarrow{r_{i}}$ and $\overrightarrow{r_{j}}$. In the approximation of isotropic oscillators, the expression for the DDD energy is analogous to Eq.(4), except that the strength coefficient is:

$$
\nu_{A A C}=\frac{3 \alpha_{A}^{2} \alpha_{C} E_{C} E_{A}\left(E_{C}+2 E_{A}\right)}{4\left(E_{A}+E_{C}\right)^{2}} .
$$

Here the energies $E_{A}$ and $E_{C}$ are characteristic energies of the adatom and $\mathrm{C}$ atom, respectively, and $\alpha_{C}$ is the static polarizability of a $\mathrm{C}$ atom 13,18 .

For simplicity, we perform the summation of gas-surface DDD interactions (10) by smearing out the carbon atoms in the substrate. This continuous approximation introduces an error, but the qualitative trends are expected to be accurate in general. Under this assumption, the net DDD contribution from three nanotubes of radius $R$ takes the form:

$$
V_{A A C}^{(3)}(z)=\frac{12 \vartheta \nu_{A A C} M(x ; y)}{z^{3} R^{4}},
$$

where $\vartheta=0.38 \AA^{-2}$ is the surface density of $\mathrm{C}$ atoms and $z$ is the distance between adatoms. Here, $x=z / R$ and $y=d / R$, where $d$ is the distance from the axis of the cylinder to the axis of the IC. The integral $M(x ; y)$ is a dimensionless integral over a cylindrical surface. Results for $V_{A A C}^{(3)}$ as a function of adatom lateral separation, in the case of quasi-1d phases of $\mathrm{He}, \mathrm{Ne}$ and $\mathrm{H}_{2}$ adsorbates, were calculated from (12) for nanotubes of radius $6.9 \AA$ and $d=9.815 \AA$.

The pair potentials employed here are those of Silvera and Goldman [19] $\left(\mathrm{H}_{2}\right)$; Aziz, Nain et al. 20] (He) and of Aziz, Meath et al. [21] (Ne). The values of DDD strength coefficient (11) were calculated from Ref. 13, 18. Results of our calculations are given in Fig. 1, Fig. 2 and Fig. 3 and in Table 1. In Table $1, \epsilon$ and $z_{m}$ are the well depth and the location of the potential minimum for $V_{\text {eff }}^{(2)} ; \epsilon_{0}$ and $z_{0 m}$ are the corresponding quantities for the free space potential; $\delta$ is the percent decrease in the well depth due to $V_{A A C}^{(3)}$ and $\rho$ is the sign-reversal point of $V_{\mathrm{eff}}^{(2)}$.

In all three cases we observe a large repulsive DDD effect: the reduction in the well depth of the pair interaction is $54 \%, 28 \%$ and $25 \%$ for $\mathrm{H}_{2}$, He and $\mathrm{Ne}$, respectively (Table 1). An interesting feature of the effective potential is the presence of a critical value of adatom separation for $\mathrm{H}_{2}$ and He adsorbates, beyond which $V_{\text {eff }}^{(2)}$ becomes repulsive for a certain range of separation values. These points are $\rho \simeq 6.3 \AA$ and $7.4 \AA$ for $\mathrm{H}_{2}$ and He, respectively (Table 1), with corresponding ranges of repulsive $V_{\text {eff }}^{(2)}: \triangle z=(6.3-15.1$ $\AA)$ and $\triangle z=(7.4-11.7 \AA)$. On the other hand, an investigation of the asymptotic behavior of $M(x ; y)$ reveals that the DDD energy becomes negative for very large interparticle separations $(z>9 R)$. We note that a reversal in sign for $V_{\text {eff }}^{(2)}$ is not found for Ne. To elucidate this fact we investigate the relative strength of DDD interaction at $z_{0 \mathrm{~m}}$. The expression (12) can be used to yield:

$$
\frac{V_{A A C}^{(3)}\left(z_{0 m}\right)}{\epsilon_{0}} \equiv k M\left(x_{o m} ; y\right)\left[\frac{\nu_{A A C} z_{0 m}^{3}}{C_{6}}\right],
$$

where $\epsilon_{0} \simeq C_{6} / 2 z^{6}$ is the free space well depth and $k$ is independent of the adsorbate species. The integral $M\left(x_{0 m} ; y\right)$ has only a weak dependence on $z_{0 m}$ in the region of interest $(3-3.5 \AA)$. Then the relatively small DDD effect for Ne is explained by the bracketed term involving $\nu_{A A C}, C_{6}$ and $z_{0 m}$ in (13). Its value is nearly twice as large for $\mathrm{H}_{2}$ as for $\mathrm{Ne}\left(34.1 \AA^{6}\right.$ vs. $\left.17.7 \AA^{6}\right)$.

The present results imply that the substrate mediation effect is approximately three times as large for the IC geometry than for adsorbed monolayer films [11]. Two factors are responsible. One is that the equilibrium distance to the substrate $(2.9 \AA)$ is $\sim 5 \%$ smaller than on a planar surface, yielding a larger substrate contribution. The more important factor is that three adjacent surfaces contribute in this IC geometry.

One significant consequence of the reduced attraction between the particles within an IC is a qualitative change in the predicted equation of state for this 1d system. It has been shown 23] that the ground state of a $1 \mathrm{~d}$ bose system is a liquid if and only if a bound dimer exists. For He, the dimer is bound by just a few milliKelvin, if the free space potential is used [24, 25, 26. There is no doubt therefore that this binding disappears when the weakened attraction is substituted. For $\mathrm{H}_{2}$, the dimer binding energy 
computed with the Silvera-Goldman potential is large $(0.384 \mathrm{meV})$ and the ground state of the system is a liquid with cohesive energy $\simeq 0.4 \mathrm{meV}$ per molecule [27]. When the new potential appropriate to the IC is used, we find no bound states. ( For $\mathrm{D}_{2}$, the dimer is very weakly bound, by $0.0513 \mathrm{meV}$ ). If our calculations are correct, they imply that the ground states of interstitial ${ }^{4} \mathrm{He}$ and para- $\mathrm{H}_{2}$ are quantum gases. Such a system is of considerable fundamental interest.

In the nanotube bundle geometry, adsorption can also occur on the outer surface of a bundle. The most favorable site energetically is the groove formed at the intersection of two external tubes, which has been studied by several groups both experimentally and theoretically. In contrast to the IC case, larger particles ( such as $\mathrm{Ar}, \mathrm{Kr}$ and $\mathrm{Xe}$ ) can fit nicely into the grooves 7 . We have computed the three-body effect of the substrate, using the DDD expression as in the IC's. The resulting effect on the pair potential for $\mathrm{H}_{2}, \mathrm{He}, \mathrm{Ne}, \mathrm{Ar}, \mathrm{Kr}$ and $\mathrm{Xe}$ is reported in Table 2. The substrate effect of reducing the well depth is smaller than in the IC case. The reasons are twofold. One is simply that only two nanotubes contribute here, instead of three in the IC case. The second reason is that the distance to the tubes is somewhat larger than in the IC case; in these grooves, the distance is very close to the $\sigma$ parameter in the gas-carbon pair interaction. (It would be exactly $\sigma$ if the tube radius were infinite). These differences parallel those used above to distinguish the IC case from the graphite case. For this reason, the groove results are intermediate between those from the latter two geometries.

\section{Three-body interactions for the $\mathrm{H}_{2}$ trimer in free space}

We have calculated the nonadditive, three-body interactions for the linear and equilateral triangle arrangements of three hydrogen molecules from first principles. The $\mathrm{H}_{2}$ bond length is kept fixed at 0.7668 $\AA$, consistent with the rigid rotor approximation. With the molecular centers-of-mass forming the vertices of a triangle, the intramolecular orientation of each molecule is described relative to three axes centered at each vertex. We have explored the effects of molecular orientation on the three-body interactions by orienting each molecule in one of three ways. Each molecule is oriented with its molecular axis perpendicular to the plane of the triangle, bisecting a corner of the triangle, or perpendicular to the line bisecting a corner. In total, there are 27 possible combinations of trimer "orientations". Due to symmetry, it is necessary to calculate only 10 non-degenerate orientations for each geometry. The closest center-of-mass to center-of-mass distance was stepped by 0.2 angstrom from 2.0 to 6.0 angstrom for the linear geometry and from 3.0 to 6.0 angstrom for the equilateral triangle arrangement.

Convergence tests were performed to determine the level of theory and basis set required for accurate calculations of three-body hydrogen interactions. We found that the coupled-clusters $(\operatorname{CCSD}(\mathrm{T})[28 \|)$ level of theory with Dunning's augmented, correlation consistent, triple zeta basis set, aug-cc-pVTZ 29] was sufficient to converge the three-body energies to within $0.1 \mathrm{~K}$. The generalized counterpoise method of Valiron and Mayer [30] was used to correct for basis set superposition error (BSSE).

In order to compare with the three-body contributions calculated using the triple-dipole approximation for isotropic oscillators (Eq.4), we have calculated isotropic potentials for both the linear and equilateral geometries. The isotropic potential is obtained by weighted averaging of the potentials representing each of the ten combinations of orientations. The weight placed on each potential is proportional to the degeneracy of the specific orientation. In effect, this corresponds to uniformly averaging over all 27 possible orientation. This is illustrated for the equilateral geometry in Fig. 4.

At close range, Hartree-Fock level contributions such as electron exchange and repulsion effects can be large [31, 32, 33]. Only at larger intermolecular separations do dispersion forces dominate. With this in mind, we calculated the Hartree-Fock nonadditive three-body potential energy surface using the same basis set and BSSE correction. A "dispersion only" three-body potential surface was approximated by subtracting the Hartree-Fock PES from the original CCSD(T) surface. Fig. 5 shows the resulting dispersion potentials for the equilateral (filled symbols) and linear (empty symbols) geometries. This approach is not a true calculation of the dispersion potential. But, the quickly decaying HF potentials suggest that the remaining interactions are mainly dispersion. Fitting the long range energies $\left(r_{\text {nearest }}\right.$ $\geq 3.8 \AA$ for the linear and $r_{\text {nearest }} \geq 4.8 \AA$ for the equilateral) using the leading triple-dipole dispersion function given by Eq. (4) resulted in a root-mean-square error of 0.013. By including the next leading 
term,

$$
U(D D Q)=Z(D D Q) \frac{9 \cos \left(\phi_{3}\right)-25 \cos \left(3 \phi_{3}\right)+6 \cos \left(\phi_{1}-\phi_{2}\right)\left[3+5 \cos \left(2 \phi_{3}\right)\right]}{r_{12}^{3} r_{23}^{4} r_{31}^{4}},
$$

one further reduces the error to 0.006 . We did not find significant improvements to the fit by including the remaining triple dispersion terms. The dispersion model fitted to both the linear and equilateral geometries are plotted in Fig. 5 as dashed and solid curves, respectively. The resulting dispersion energy constants are $Z(D D D)=49607 \mathrm{~K} \cdot \AA^{9}$ and $Z(D D Q)=5763 \mathrm{~K} \cdot \AA^{11}$. McDowell and Meath report an "exact" value for $Z(D D D)$ of $49833 \mathrm{~K} \cdot \AA^{9}$, calculated from dipole oscillator strength distribution data [34]. McDowell[17] observed that for a several specific orientations of $\mathrm{H}_{2}$ trimers (all three molecules oriented in the same plane), nonadditive induction contributions can be as large as those from DDD. This, however, is not apparent in the isotropic potentials, where the induction effects have been completely "washed-out" by the uniform angle-averaging process [32], as is the case with permanent multipole interactions.

The Hartree-Fock potentials in Fig. 5 indicate that non-dispersion interactions, such as exchange/repulsion effects, are substantial at short range. We will present a thorough analysis of the different origins of hydrogen three-body interactions in a forthcoming article [35]. It is clear from the present study that the DDD approximation, alone, is not sufficient at describing the total $(\operatorname{CCSD}(\mathrm{T}))$ three-body interactions at short distances. Whether this hold true for systems containing the other constituents remains unclear and would require separate $a b$ initio calculations.

\section{Discussion}

In this paper we have found that three-body interactions play an important role in altering the interactions between adatoms within IC's between carbon nanotubes. The reduction in the well depth of the pair interaction is $54 \%, 28 \%$ and $25 \%$ for $\mathrm{H}_{2}$, $\mathrm{He}$ and $\mathrm{Ne}$, respectively. The magnitude of these shifts is roughly similar to the result of a crude estimate, i.e. a factor of three increase over the effect of a single planar graphite surface. The DDD term even becomes larger in magnitude than the two-body potential for interparticle separations above $~ 7.4 \AA$ and $6.3 \AA$ for $\mathrm{He}$ and $\mathrm{H}_{2}$, respectively. Such a large DDD effect implies a significant change in the condensation properties of $\mathrm{H}_{2}$ and $\mathrm{He}$ in carbon nanotube bundles. For $\left(\mathrm{H}_{2}\right)_{2}$ the reduction in well depth is sufficient as to destroy the bound state. The perimeter of a nanotube bundle is accessible for adsorbate particles of bigger size. In our approach, the observed reduction in the well depth of the pair interaction due to DDD effect is still significant: $35 \%, 24 \%, 15 \%, 19 \%, 22 \%$ and $28 \%$ for $\mathrm{H}_{2}, \mathrm{He}, \mathrm{Ne}, \mathrm{Ar}, \mathrm{Kr}$ and Xe, respectively.

The important consequence from the DDD effect for quasi- $1 \mathrm{~d}$ IC phase of $\mathrm{H}_{2}$ molecules and $\mathrm{He}$ atoms is that there is no dimer bound state. The lack of the latter implies that there is no bound state for the $N$ particle system. Then, the ground state of $\mathrm{H}_{2}$ molecules interacting with the screened pair potential is a gas, not a liquid. This result raises an interesting question: what is the low temperature behavior of such a marginally unbound system?

Finally, ab initio calculations of three-body interactions for linear and equilateral triangle arrangements of three $\mathrm{H}_{2}$ molecules provide implications for the behavior of three-dimensional phases of hydrogen. Moreover, they justify a future $a b$ initio study of the exchange interaction of two $\mathrm{H}_{2}$ molecules and a carbon atom within a nanotube.

We are grateful to Keith Williams, Paul Sokol, Peter Eklund and Moses Chan for helpful discussions. This research has been supported by the Army Research Office under grant DAAD 19-99-0167. 


\section{References}

[1] Q.Wang, S.R.Challa, D.S.Sholl, and J.K.Johnson, Phys.Rev.Lett. 82, 956 (1999).

[2] S.Inoue, N.Ichikuni, T.Suzuki, T.Uematsu, and K.Kaneko, J.Phys.Chem. B, 102, 4689 (1998).

[3] W.Teizer, R.B.Hallock, E.Dujardin, and T.W.Ebbesen, Phys.Rev.Lett. 82, 5305 (1999), E84, 1844 (2000).

[4] S.E.Weber, S.Talapatra, C.Journet, A.Z.Zambano and A.D.Migone, Phys.Rev. B 61, 13150 ( 2000 ).

[5] A.Kuznetsova, J.T.Yates,Jr., J.Liu, and R.E.Smalley, J. Chem. Phys. 112, 9590 (2000).

[6] K.A.Williams and P.C.Eklund, Chem.Phys.Lett. 320, 352 (2000).

[7] G.Stan, M.J.Bojan, S.Curtarolo, S.M.Gatica and M.W.Cole, "Uptake of gases in bundles of carbon nanotubes", to appear in Phys.Rev.

[8] M.W.Cole, V.H.Crespi, G.Stan, J.M.Hartman, S.Moroni, and M.Boninsegni, Phys.Rev.Lett. 84, 3887 (2000).

[9] M.J.Elrod and R.J.Saykally, Chem.Reviews 94, 1975 (1994).

[10] J.A.Barker, in "Simple Molecular Systems at Very High Density", ed.A.Polian, P.Loubeyre and N.Boccara, Plenum, NY,(1989), pp. 341-351 .

[11] See Chapt.2 and appendix E of L.W.Bruch, M.W.Cole and E.Zaremba, "Physical Adsorption: Forces and Phenomena" (Oxford U.P., 1997).

[12] A.D.McLachlan, Mol. Phys. 7, 381 (1964).

[13] S.Rauber, J.R.Klein, M.W.Cole and L.W.Bruch, Surf. Sci. 123, 173 (1982).

[14] J.M.Gottlieb and L.W.Bruch, Phys.Rev. B 48, 3943 (1993).

[15] P. Diep and J. K. Johnson, J. Chem. Phys. 112, 4465 (2000).

[16] L.M.Woods and G.D.Mahan, Phys. Rev. B 61, 10651 (2000).

[17] S.A.C.McDowell, J.Chem.Phys. 105, 4180 (1996).

[18] J.R.Klein, L.W.Bruch, M.W.Cole, Surf.Sci. 173, 555 (1986).

[19] I.F.Silvera and V.V.Goldman, J. Chem. Phys. 69, 4209 (1978).

[20] R.A.Aziz, V.P.S.Nain, J.S.Carley, W.L.Taylor, G.T.McConville, J.Chem.Phys. 70, 4330 (1979).

[21] R.A.Aziz, W.J.Meath, A.R.Allnatt, Chem. Phys. 78, 295 (1983).

[22] R.A.Aziz, in "Inert Gases", ed. M.L.Klein, Springer-Verlag (1984), appendix 2.B .

[23] E.Krotscheck, M.D.Miller and J.Wojdylo, Phys.Rev. B 60, 13028 (1999).

[24] G.Stan, M.Boninsegni, V.Crespi, and M.W. Cole, J. Low Temp. Phys. 113, 447 (1998).

[25] M.Boninsegni, S.Moroni, J. Low Temp. Phys. 118, 1 (2000).

[26] M.C.Gordillo, J.Boronat, and J.Casulleras, Phys. Rev. B 1, R878 (2000).

[27] M.C.Gordillo, J.Boronat, and J.Casulleras, to be published in J. Low Temp. Phys.

[28] J. Gauss, J.F. Stanton, and R.J.Bartlett, J. Chem. Phys. 95, 2639 (1991).

[29] T.H.Dunning, Jr., J. Chem. Phys. 90, 1007 (1989). 
[30] P.Valiron and I.Mayer, Chem. Phys. Lett. 275, 46 (1997).

[31] P.Rosen, J. Chem. Phys. 21, 1007 (1953).

[32] V.F.Lotrich and K.Szalewicz, J. Chem. Phys. 106, 9688 (1997).

[33] J.Higgins, T.Hollebeek, J.Reho, T.S.Ho, K.K.Lehmann, H.Rabitz, and G.Scoles, J. Chem. Phys. 112, $5751(2000)$.

[34] S.A.C.McDowell and W.J.Meath, Mol. Phys. 90, 713 (1997).

[35] P.Diep and J.K.Johnson, "Three-body Interactions of Hydrogen from First Principles", in preparation. 


\section{Tables}

Table 1: DDD effect for $\mathrm{H}_{2}$, He and Ne adsorbates confined within IC's of a nanotube bundle. All lengths are in $\AA$ units; $\epsilon$ and $\epsilon_{0}$ are in $\mathrm{meV}$.

\begin{tabular}{|c|c|c|c|}
\hline Quantity & $\mathrm{H}_{2}$ & $\mathrm{He}$ & $\mathrm{Ne}$ \\
\hline$\epsilon\left(\epsilon_{0}\right)$ & $1.35(2.96)$ & $0.67(0.93)$ & $2.72(3.64)$ \\
$\delta(\%)$ & 54 & 28 & 25 \\
$z_{m}\left(z_{0 m}\right)$ & $3.55(3.41)$ & $3.00(2.97)$ & $3.12(3.09)$ \\
$\rho$ & 6.3 & 7.4 & - \\
\hline
\end{tabular}


Table 2: DDD effect for $\mathrm{H}_{2}, \mathrm{He}, \mathrm{Ne}, \mathrm{Ar}, \mathrm{Kr}$ and Xe adsorbates confined into the groove of a nanotube bundle. All lengths are in $\AA$ units; $\epsilon$ and $\epsilon_{0}$ are in $\mathrm{meV}$. The free space potential parameters for $\mathrm{Ar}, \mathrm{Kr}$ and Xe were taken from Ref. [22].

\begin{tabular}{|c|c|c|c|c|c|c|}
\hline Quantity & $\mathrm{H}_{2}$ & $\mathrm{He}$ & $\mathrm{Ne}$ & $\mathrm{Ar}$ & $\mathrm{Kr}$ & $\mathrm{Xe}$ \\
\hline$\sigma$ & 2.97 & 2.74 & 3.00 & 3.40 & 3.42 & 3.40 \\
$\epsilon\left(\epsilon_{0}\right)$ & $1.93(2.96)$ & $0.71(0.93)$ & $3.08(3.64)$ & $10.1(12.3)$ & $13.5(17.2)$ & $17.5(24.2)$ \\
$\delta(\%)$ & 35 & 24 & 15 & 19 & 22 & 28 \\
$z_{m}\left(z_{\text {om }}\right)$ & $3.49(3.41)$ & $3.00(2.97)$ & $3.11(3.09)$ & $3.79(3.76)$ & $4.05(4.01)$ & $4.42(4.36)$ \\
\hline
\end{tabular}




\section{Figure Captions}

Fig.1. Free-space potential $U_{A A}^{2}$ (circle/full curve), the DDD potential $V_{A A C}^{(3)}$ (triangle/full curve) and the effective pair potential $V_{\text {eff }}^{(2)}$ (solid curve) for $\mathrm{H}_{2}$ molecules adsorbed within the IC's of nanotube bundles are shown. The inset shows the behavior of $V_{\mathrm{eff}}^{(2)}$ close to the sign-reversal point.

Fig.2. Same as Fig.1 for He.

Fig.3. Same as Fig.1 for Ne. The inset shows that there is no reversal in sign for $V_{\mathrm{eff}}^{(2)}$ in this case.

Fig.4.Angle-averaging the 27 orientations of the equilateral triangle arrangement of $\left(\mathrm{H}_{2}\right)_{3}$ to obtain an isotropic potential, show in the bold curve with filled circles. The thin curves represent each of the 10 distinct orientations at each separation.

Fig.5 .Hartree-Fock plus triple-dispersion fit of equilateral and linear isotropic potentials. Calculated data are represented in symbols (filled $=$ equilateral, empty $=$ linear). Solid curves denote fits to equilateral geometry; dashed curves are fits to linear geometry. 


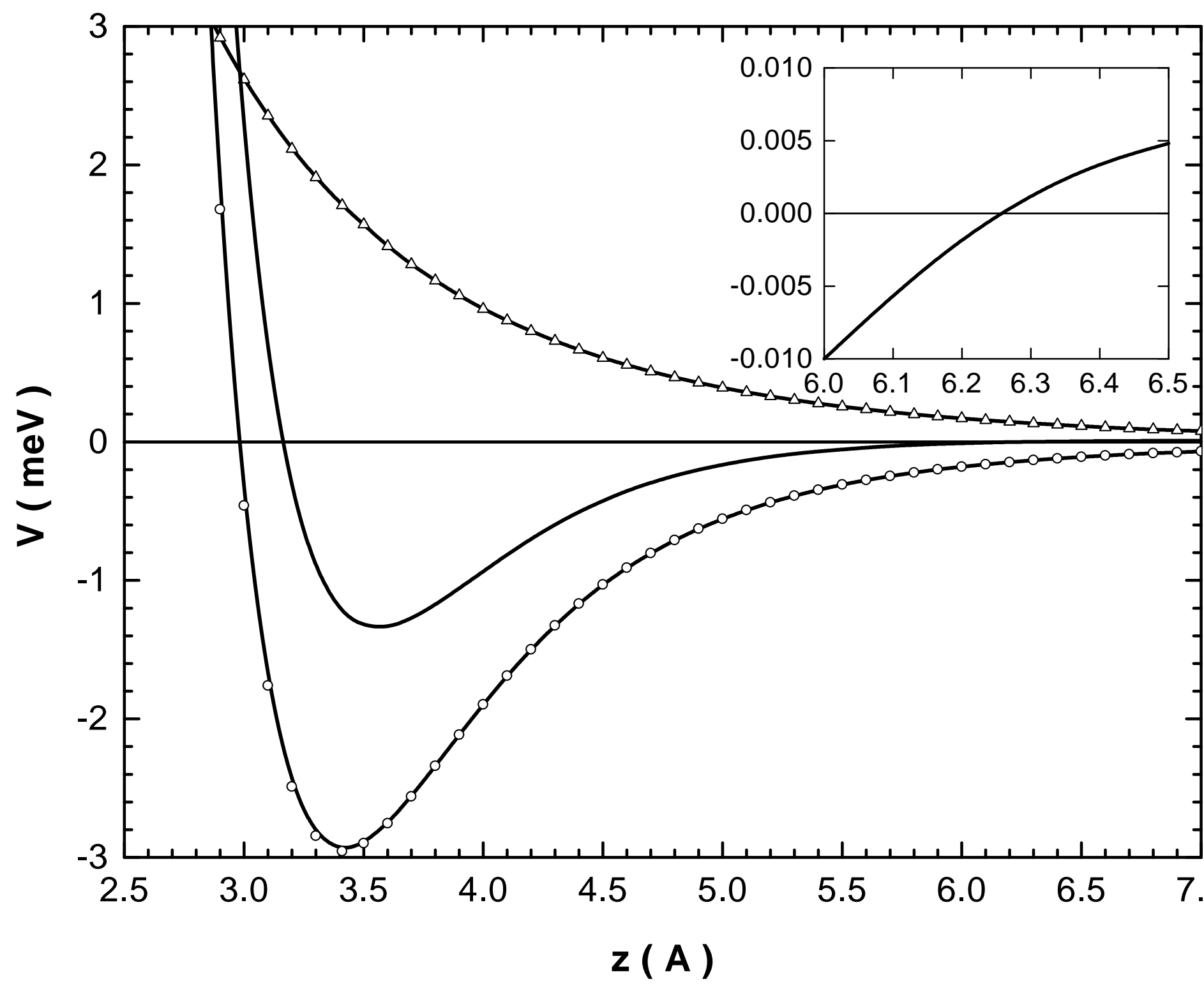

Fig.1 


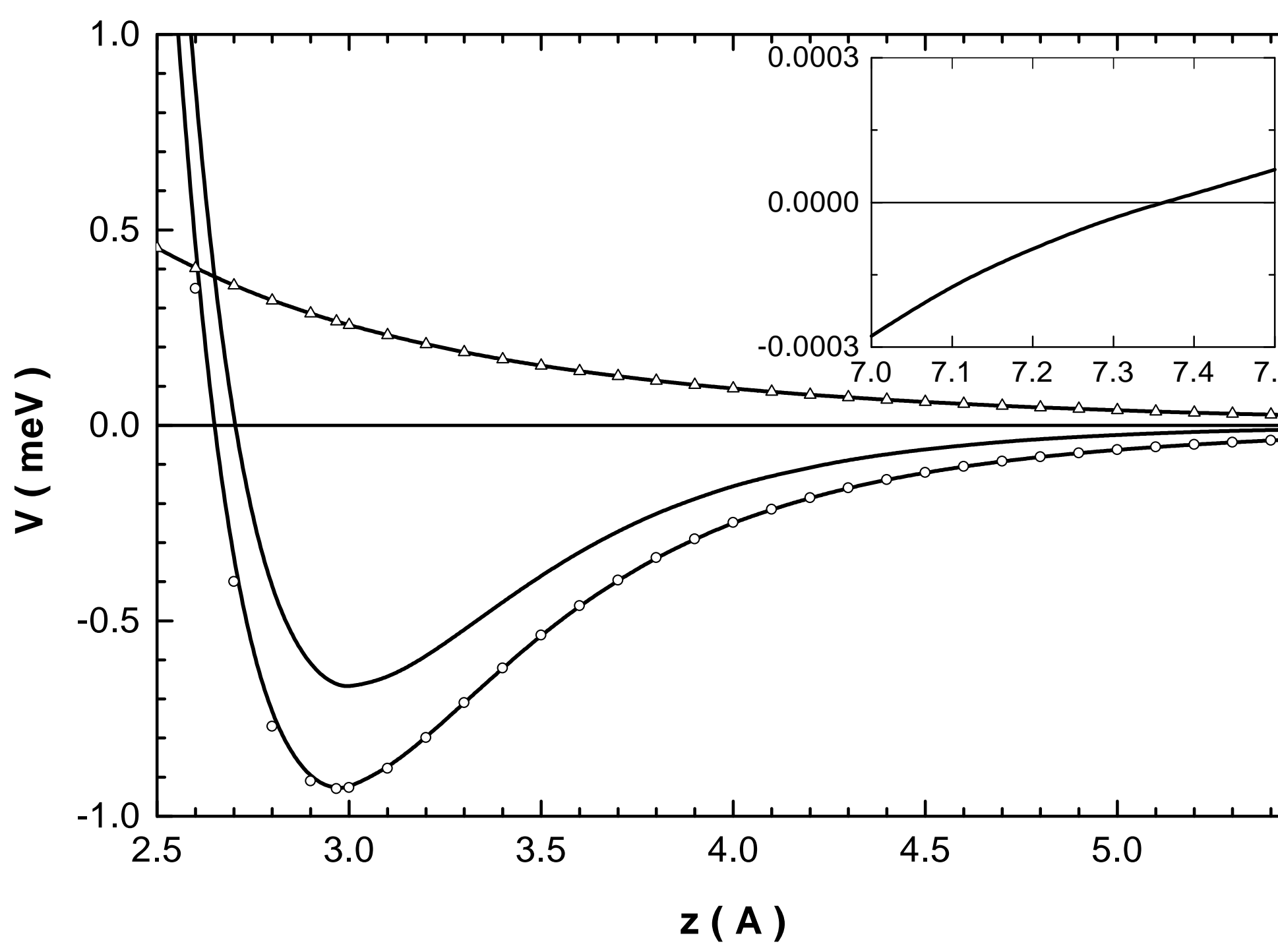

Fig.2 


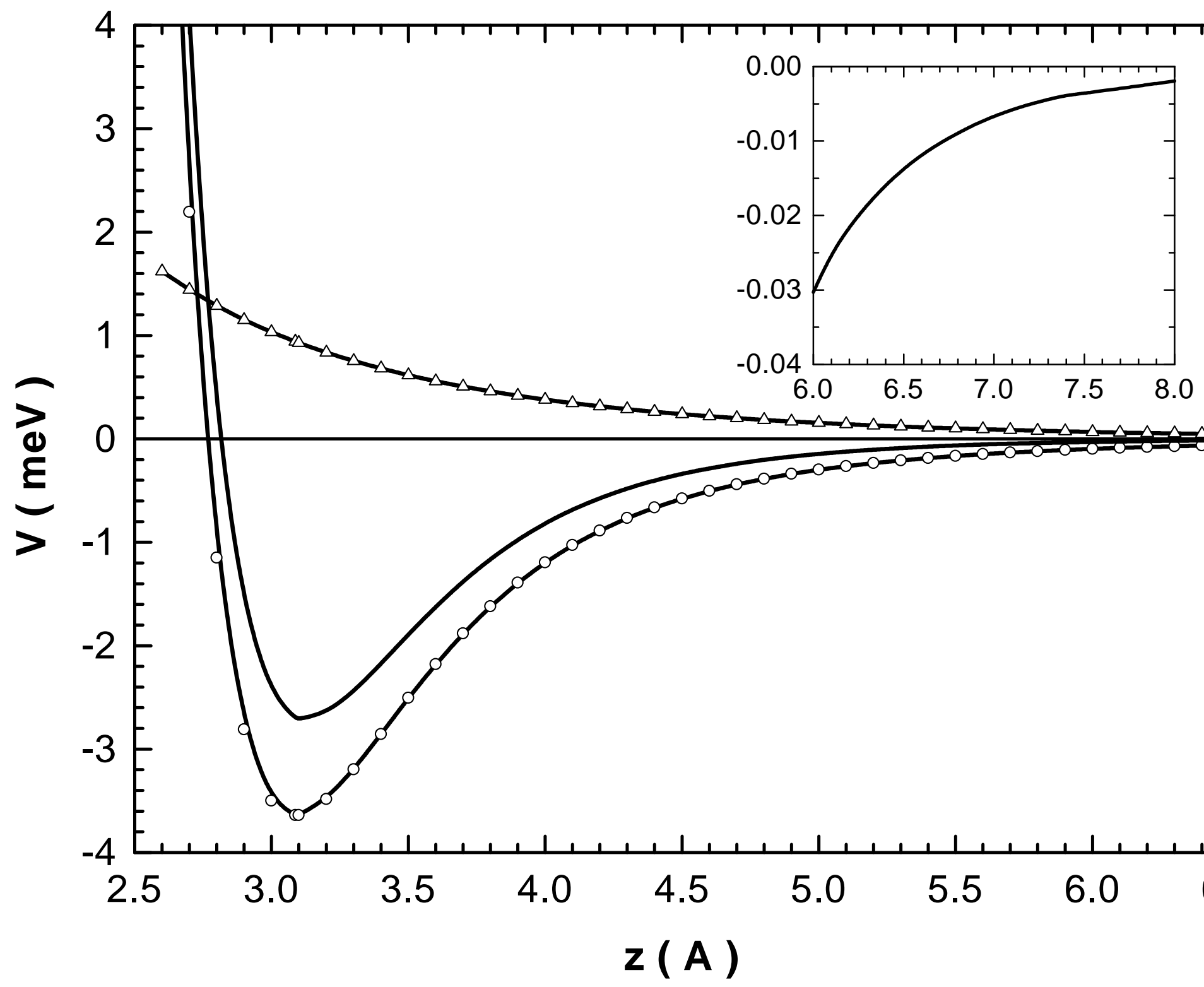

Fig.3 


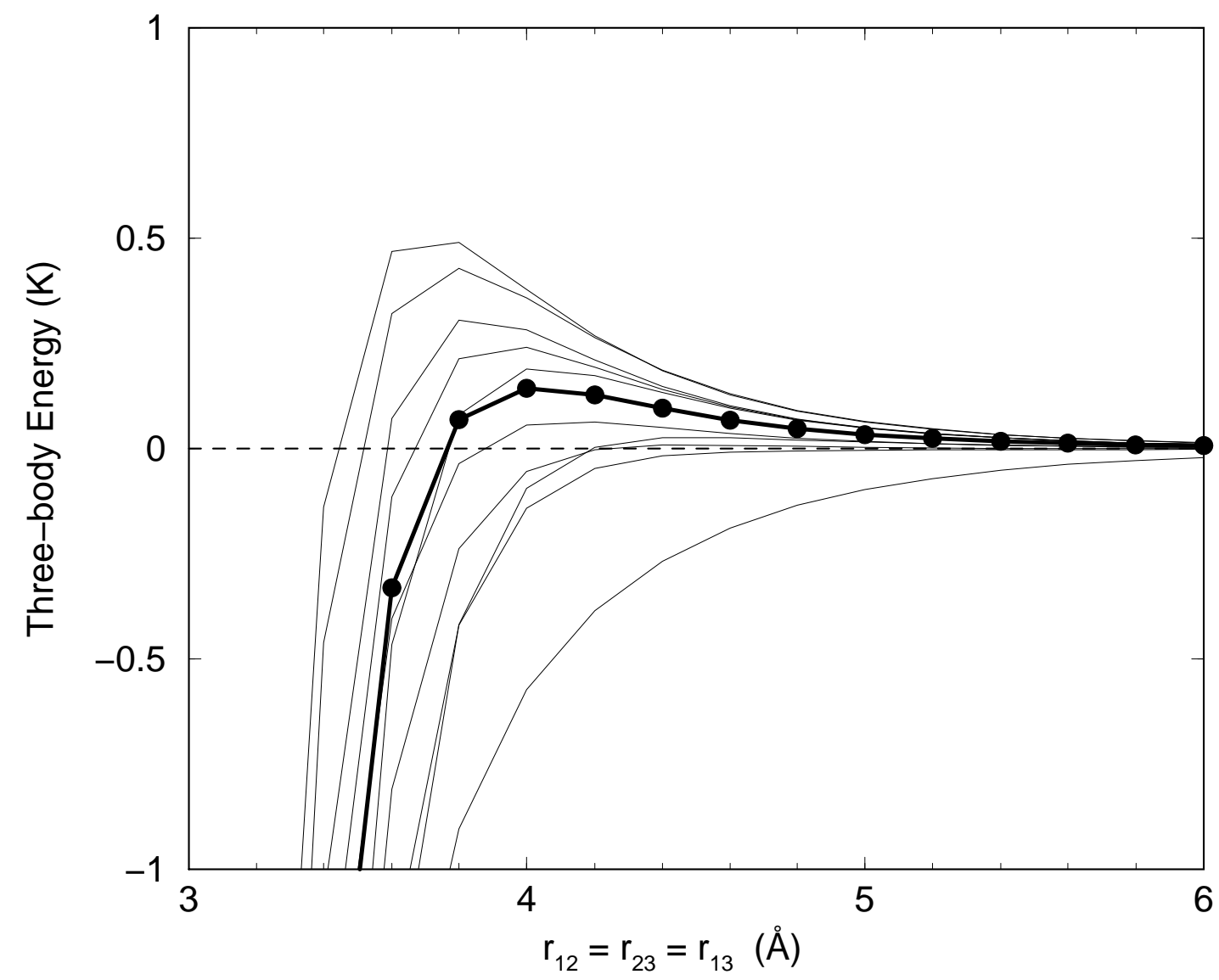

Fig. 4 


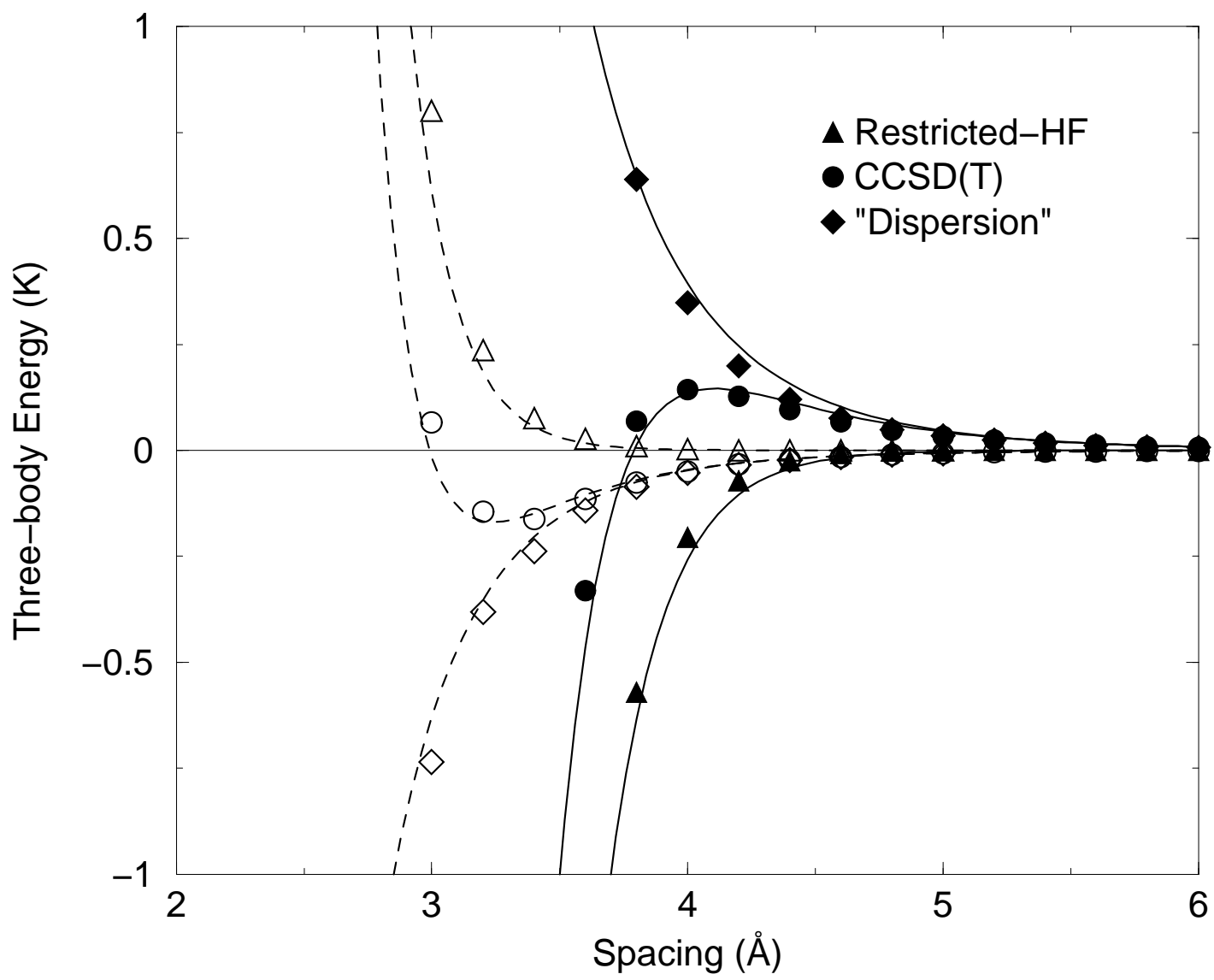

Fig. 5 